\title{
Reflexiones sobre el proceso de investigación cualitativa: una experiencia de tra- bajo acerca del empoderamiento de las mujeres y el conflicto armado
}

\section{Reflections on the qualitative research process: a work experience about the em- powerment of women and the armed conflict}

\author{
Yolima Amado Sánchez ${ }^{1}$ \\ Martha Elena Saboyá Ramírez ${ }^{2}$ \\ DOI: 10.29151/hojasyhablas.n17a6
}

\section{Resumen}

Este artículo tiene como propósito presentar algunos de los análisis derivados de la experiencia investigativa realizada durante los años 2016 y 2017, en el marco del proyecto de investigación titulado "Procesos de redefinición del rol social femenino a la luz de la ética de género", en tres regiones de Colombia, con nueve organizaciones sociales de mujeres. En esta ocasión, y fundamentalmente con objetivos pedagógicos, presentamos algunas reflexiones que surgieron en el marco del quehacer investigativo, en tres momentos diferentes: en retrospectiva, en la mirada puntual sobre los dos años que duró la investigación y en prospectiva. El trabajo significó para las autoras el encuentro con situaciones y circunstancias inesperadas que reclamaron una atención que trascendió el cumplimiento de un cierto plan de investigación, en procura del reconocimiento de la complejidad de las situaciones de las participantes; un ejercicio del que se derivó un cuestionamiento ético acerca del lugar de la investigación, de la academia y de la sociedad civil, ante las complejidades de la realidad social colombiana.

Palabras clave: Investigación cualitativa; producción escrita; subjetividad; mujeres; conflicto armado.

\begin{abstract}
The purpose of this article is to present some of the analyzes derived from the research experience carried out during the years 2016 and 2017, within the framework of a research project entitled: "Processes of redefining the female social role, in light of gender ethics", in three regions of Colombia, with nine women's social organizations. On this occasion, and fundamentally with pedagogical objectives, we present some reflections that emerged within the framework of the investigative work, in three different moments: in retrospect, in the point of view on the two years that the research lasted and in prospective. The work, meant for the authors the encounter with unexpected situations and circumstances that demanded attention for a certain research plan, in search of recognition in the middle of complex situations of the participants; an exercise that led to an ethical questioning about the place of research, academia and civil society, given the complexities of Colombian social reality.
\end{abstract}

Keywords: Qualitative research; written production; subjectivity; women; armed conflict.

\footnotetext{
${ }^{1}$ Magister en Psicoanálisis, Subjetividad y Cultura y Psicóloga de la Universidad Nacional de Colombia; docente universitaria e investigadora, autora del libro ¡Todos a-formar! La producción escrita de los estudiantes universitarios: Los obreros del Alma Mater y de varios artículos en revistas científicas del país. Profesora de tiempo completo del Instituto de Estudios en Familia, de la Fundación Universitaria Monserrate-Unimonserrate. Correos electrónicos: yamados@unimonserrate.edu.co, yamados@unal.edu.co. ORCID: https:// orcid.org/0000-0001-9228-8002

${ }^{2}$ Magister en Política Social de la Universidad Externado de Colombia y Trabajadora Social de la Universidad Nacional de Colombia. Docente universitaria e investigadora, autora de varios artículos en revistas científicas del país. Profesora de la Corporación Universitaria Minuto de Dios, UNIMINUTO, Regional Zipaquirá. Correo electrónico: masara16@ hotmail.com. ORCID: https://orcid.org/0000-0002-9331-8813
} 


\section{Los tiempos del proceso de investigación}

"Hemos adquirido conocimientos sin precedentes sobre el mundo físico, biológico, psicológico, sociológico. La ciencia ha hecho reinar, cada vez más, a los métodos de verificación empírica y lógica. Mitos y tinieblas parecen ser rechazados a los bajos fondos del espíritu por las luces de la Razón. $Y$, sin embargo, el error, la ignorancia, la ceguera, progresan, por todas partes, al mismo tiempo que nuestros conocimientos" (Morin, 2009)

El presente artículo obedece a la intención de aportar elementos de análisis para el ejercicio formativo en investigación, especialmente, en el caso de quienes nos ocupamos de la investigación de fenómenos sociales, que las más de las veces, implican el encuentro con circunstancias, realidades, complejidades y fenómenos más allá de los previstos durante el frío periodo de la planeación del proceso. En nuestro caso, asumir el desarrollo del proyecto obedeció a la asignación de una responsabilidad vinculada con las tareas propias de la distribución del tiempo contractual en las diferentes funciones sustantivas, en nuestra calidad de docentes universitarias ${ }^{3}$.

Presentaremos el recuento de tres momentos que resultaron cruciales a lo largo del proceso de investigación desarrollado por las autoras, pues implicaron la toma de decisiones, la realización de ajustes en momentos no planeados, el reconocimiento de contradicciones, la confrontación con las posturas personales y profesionales, y el reclamo ético suscitado en el encuentro mismo con las comunidades, propio de la etapa del trabajo de campo; así mismo, buscamos compartir algunas particularidades derivadas de las conversaciones que realizamos, como producto del acercamiento a las realidades sociales encontradas, en contraste con los puntos de vista sobre el papel de la investigación, de cara a la docencia y a la construcción de sociedad.

Igualmente, nos interesa destacar el papel que cumple la investigación social cualitativa, como ese giro en la mirada que constituye aquel "retorno al sujeto" que menciona Galeano (2018), y que nos brinda la posibilidad de adentrarnos en el vasto, dinámico y cambiante universo de lo "social", aunada a cierta demanda por contribuir al proceso de la reivindicación de las ciudadanías y a la construcción de conocimientos diversos, contrastantes, que aporten a la revisión y visibilización de los procesos sociales, en la perspectiva de aportar reflexiones y argumentos que posicionen debates tendientes a desnaturalizar las situaciones de inequidad e indignidad que padecen nuestras compatriotas, desde el reconocimiento de la corresponsabilidad que tenemos, como parte de la sociedad civil.

Decidimos construir el artículo considerando tres momentos, por cuanto nos permitirá, a manera de línea de tiempo, identificar el recorrido de cara a algunas consideraciones propias de los contextos académicos y extraacadémicos que nos han venido impactando a lo largo de nuestra formación profesional, para luego pasar a identificar los aspectos formales dentro de los cuales se proyectó la investigación y los aprendizajes obtenidos, tanto en el terreno personal como profesional,

\footnotetext{
${ }^{3}$ El proyecto en cuestión, fue financiado durante los años 2016 y 2017 por la Fundación Universitaria Cervantina San Agustín -Unicervantina, actualmente denominada Fundación Universitaria Unicervantes. El proceso se truncó, por la terminación de los contratos de las autoras y por la posterior decisión de no renovarlos, por parte de las directivas de la institución universitaria.
} 
durante la ejecución y, finalmente, realizar apuntes -a manera de proyección- sobre el para qué de la investigación.

\section{En retrospectiva}

Las docentes investigadoras fueron formadas por la Universidad Nacional de Colombia, con diferencia aproximada de veinticinco años entre una y otra. Una de ellas avizoró su interés por formarse en el pregrado en Trabajo Social en los años 70, época que para la universidad pública fue de gran convulsión estudiantil en el país, por cuestionamientos que se adelantaban en torno a asuntos internos, pero igualmente a asuntos externos. Respecto a los internos el gran debate surgía por la consabida reforma educativa, que propugnaba por la tecnologización de la educación y la ampliación de cobertura en los niveles de educación básica, media y superior, pero sin contar con estrategias o recursos que fueran garantes de que la cantidad no sacrificara la calidad. Desde luego, aparecían otros temas de interés nacional, como el auge de la producción marimbera para el mercado internacional y su impacto en la producción nacional, al lograr desplazar cultivos alimenticios, a su vez el consumo interno empieza a apreciarse y en los medios universitarios y a usarse, por algunos, como inhibidor de tensiones.

Eran evidentes, con mayor o menor resonancia, los conflictos regionales por las luchas que el Estado libraba en contra de organizaciones políticas al margen de la ley las cuales en su momento reivindicaban la justicia social, precepto considerado válido por el conglomerado estudiantil, que, además, debatía acerca de la propiedad de la tierra y la necesidad de defensa y apoyo al movimiento campesino e indígena. Respecto a los contextos externos, la comunidad educativa se interesaba por cuestionarse sobre la guerra que Occidente, pero específicamente, que Estados Unidos sostenía en el continente asiático en contra de Vietnam y los desastres humanos que se derivaban del uso del agente naranja, químico que neutralizaba de manera devastadora a nacionales asentados en esos territorios, pero también, a los mismos combatientes occidentales, al ocasionar muerte, mutilación, malformaciones genéticas, deforestación y contaminación ambiental.

En lo concerniente a la institucionalidad universitaria, ocurrió el cierre de varios programas formativos en ciencias humanas y sociales en universidades privadas, y el "vuelo" al monte de algunos docentes y estudiantes que creyeron en la transformación radical de la sociedad y los cambios a favor de los pobres mediante la acción armada. Al interior del programa académico de Trabajo Social en los años 70, "se da inicio a expresiones de un movimiento académico y político que intentaba renovar los encuadres filosóficos, teóricos y metodológicos de la profesión, conocido como La Reconceptualización” (Leal y Malagón, 2006, p.3), esto es, de cuestionamiento al contenido de los planes curriculares que contaban con una estructura que gravitaba de manera sostenida en el funcionalismo. Se polemizaba en torno a la cuestión de la investigación científica, a la importancia de la cuantificación como elemento consustancial para lograr objetividad, principalmente en apego al positivismo con sus variados enfoques y, desde luego, a la búsqueda de la pretendida cientificidad mediante la adopción, para las ciencias sociales, de las metodologías y esquemas propios de las ciencias básicas.

El énfasis, por esos años, de los trabajos investigativos se centraba en los estudios narrativos, descriptivos, comparativos todos ellos, con intención de preservar "la objetividad" poniendo distancia del objeto investigado, evitando el debate 
sobre lo que durante mucho tiempo se postuló como principio, la llamada "neutralidad valorativa”, propuesta por Max Weber. Con el avance de los años y los debates sobre el tema, se abren paso planteamientos que cuestionan el paradigma dominante: "Todo proceso de conocimiento (y por supuesto de conocimiento científico) tiene su raíz en opciones alternativas que adoptamos según juicios de valor previamente establecidos [...], juicio de valor es a menudo la selección del problema a estudiar e incluso de las hipótesis que se fabrican, juicio de valor existe en la selección de variables". (Prats, 2011, p.14); sin embargo, esto no significa que el producto final de nuestra actividad no pueda dar como resultado un conocimiento objetivo, pues las investigadoras sociales somos capaces de actuar en base a la racionalidad y los juicios de valores podrán quedar circunscritos a las opiniones personales, sin que los mismos desdibujen las reflexiones derivadas del proceso o limiten el encuentro con las y los participantes.

La otra docente, formada en el programa de Psicología a partir de los últimos años de la década del 90, ingresó a la universidad en las épocas convulsas que sobrevinieron a la Constitución del 91, signadas principalmente por hechos violentos derivados de los embates del fortalecimiento del narcotráfico en el país y las constantes noticias acerca de las tomas guerrilleras y las confrontaciones armadas. No obstante, su vinculación a la educación superior se desarrolló casi al margen de las reflexiones y análisis concomitantes con la realidad del país. Los contenidos de las asignaturas estaban dirigidos a la formación en torno a corrientes, autores, datos y contenidos que, en apariencia, parecían desligados del acontecer nacional, salvo en el caso de algunas asignaturas electivas que se acercaban a asuntos tales como el proceso de reincorporación a la vida civil de ex combatientes.
El quehacer investigativo, por llamarle de algún modo, concernía a la lectura de textos solicitados por docentes y la posterior escritura de reseñas y ensayos que, en algún sentido, resultaban ser mero parafraseo; las evaluaciones, por su parte, buscaban establecer qué tanto de lo planteado en las clases era susceptible de ser repetido, en un ejercicio mnemotécnico, que parecía situar a autores y sus obras, como poseedores de las explicaciones $\mathrm{y}$, por tanto, a los saberes como inmutables y estáticos. El ejercicio de análisis de las cuestiones contemporáneas no parecía ser relevante en las aulas, empero, fuera de ellas, en los pasillos se sostenían y propiciaban debates sobre las más variadas cuestiones, tanto aquellas que tenían que ver con el proceso de continuada intención de reforma de la Universidad, los reparos provenientes de la posible privatización de la universidad pública, la movilización de la ciudadanía en favor de algunas causas sociales, así como con la reflexión sobre la complejidad política, económica y social del país y de Latinoamérica, en términos generales.

Los actores en conflicto eran reconocidos como bandos disímiles, las guerrillas, los paramilitares, los grupos narcotraficantes y el Estado, sin embargo, eran solo algunos quienes se tomaban la tarea de analizar a profundidad los fenómenos, de reconocer las historias. Para los demás, en la ciudad y más aún, en el campus, tales realidades habían pasado a ser parte del espectáculo del país, que se alternaban con las emociones derivadas de la participación de la selección Colombia en los mundiales de fútbol, y los efectos de la ampliación de la franja televisiva tras la creación de los canales privados de televisión. Se esperaba con ansia el cambio de siglo y los avances tecnológicos que traerían nuevas opciones de acceso a la información y las comunicaciones. Los trabajos escritos dejaron de ser mecanografiados en máquinas de escribir, se imponía el uso de los ordenadores, y 
con ellos, de los motores de búsqueda en la red.

Luego, un evento internacional atenazó las perspectivas de análisis, los hechos del 11 de septiembre de 2001 en Estados Unidos de América, la discusión sobre el terrorismo, el "golpe" a ese país, y la nimiedad de unas muertes respecto a otras. La movilización social, tanto en la universidad como fuera de ella, bien pronto empezó a ser estigmatizada como acto "terrorista" y la zozobra provenida del norte del continente, ambientada con imágenes y videos de los ataques que se repetían una y otra vez, se expandió entre la ciudadanía. La necesidad de garantizar la seguridad, junto a la reverberación de los miedos fruto del copioso intercambio de información de toda índole y grado de veracidad en la red, incrementó la distancia entre las realidades de la pantalla y las de la cotidianidad.

Aun así, podríamos afirmar que el efecto no fue de sensibilización respecto a la complejidad social, más bien de saturación y de indistinción, "indiferencia por saturación, información y aislamiento" (Lipovetsky, 2000, p. 44), los medios de divulgación de información se diversificaron pronto, así como las adaptaciones tecnológicas para la comunicación, y los hechos o sucesos del mundo empezaron a divulgarse por diferentes vías, haciendo incluso de las muertes de los congéneres, un espectáculo más en el escaparate del entretenimiento.

En cuestión de apenas ocho o diez años la vida social en nuestro país, así como las posibilidades del acceso a la información, fueron drásticamente afectadas por la globalización de los gadgets tecnológicos. Y mientras en Occidente el recrudecimiento de las acciones bélicas contra el "terrorismo" eran televisadas y retransmitidas, como si se tratara de un ejercicio ejemplarizante, en el país se imponía en el imaginario social la necesidad de la "seguridad" ante el "terrorismo" doméstico, a la par de la diversificación de las opciones del entretenimiento y de la necesidad de proteger lo propio,

\begin{abstract}
Mass-mediática del aumento de la violencia en el mundo moderno: la guerra está a nuestras puertas, vivimos sobre un barril de pólvora, fíjense en el terrorismo internacional, los crímenes, la inseguridad en las ciudades, la violencia racial en las calles y en las escuelas, los atracos, etc." (Lipovetsky, 2000, p. 69).
\end{abstract}

Una individuación "necesaria” que empujaba los propósitos de cada quien hacia la inmediatez, y que, en términos de la producción del conocimiento, parecía sostenerse en el imperativo de la pululación de artículos e investigaciones sobre los más diversos asuntos, ahora, supeditados a los estándares internacionales, a los criterios de la sociedad del conocimiento que se acompasó al tenor de la masificación y proliferación de las tecnologías de la información y la comunicación.

Ahora bien, a lo largo de este periodo de tiempo que nos hemos permitido caracterizar brevemente, atendiendo a los momentos de formación profesional de las investigadoras, es preciso señalar que la pretendida objetividad de las ciencias básicas intentaba imponerse en los escenarios de la investigación social y se reiteraba la necesaria distancia que debía asumirse respecto del investigado, en aras del conocimiento científico.

Para el grupo en el que se ubican el positivismo y el pospositivismo, es esencial que el investigador adopte una postura distante y no interactiva como condición de rigor, que permita excluir los juicios valorativos o cualquier otra influencia derivada de la visión propia, tanto del investigador 
como de los sujetos objeto de investigación, de los análisis e interpretaciones, que dan origen a los resultados y las conclusiones de la investigación (Sandoval, 1996, p. 29).

Muy seguramente, si se realizara una revisión documental de los trabajos investigativos realizados durante las décadas del 70 y 80 , incluso 90, se encontrarían tesis con pretensión de establecer generalizaciones sobre la realidad social o de explicar hechos con la pretendida intención de inferir que lo investigado en unas latitudes puede encontrarse en igualdad de condiciones en otras. Un modelo que, hemos de reconocer, se articulaba con las disposiciones que, en materia económica y de política pública, estaban siendo definidas para el país en su conjunto, es decir, provenientes de las políticas aperturistas y el modelo neoliberal, dependiente de las formulaciones y corrientes intelectuales extranjeras y de los lineamientos de los organismos multilaterales (Castañeda, 2002, p. 111), tanto en términos de su proyección social, como de la proyección de su producción investigativa.

En la última década del siglo pasado y la primera del presente siglo, acompasado con el fenómeno de la globalización emergieron nuevas miradas y posturas epistemológicas que cuestionan el entramado tradicional de acercamiento a la realidad, el cual históricamente pretendió abordar las realidades mediante miradas reduccionistas, simplificantes y en desarticulación entre sus variados componentes. Entendimos cada vez más $y$, gracias a estudiosos como Morin (2009), que el estudio de cualquier aspecto de la experiencia humana ha de ser, por necesidad, multifacético; que el acercamiento a las realidades sociales sólo es posible en conexión con tradiciones familiares, sociales, genéricas, étnicas, raciales, contextos, certidumbres relativas e incertidumbres siempre presentes.

Mientras que el pensamiento simplificador desintegra la complejidad de lo real, el pensamiento complejo integra lo más posible los modos simplificadores de pensar, pero rechaza las consecuencias mutilantes, reduccionistas, unidimensionalizantes y finalmente cegadoras de una simplificación que se toma por reflejo de aquello que hubiere de real en la realidad (Morin, 2009, p. 11).

Nuestra manera de acercarnos a la realidad sin duda incorporó estos planteamientos, en el entendido que sólo podremos comprender lo concreto y específico en la diversidad, esto es, en la mirada necesaria al conjunto de elementos imbricados en los contextos, así como a las relaciones entre ellos.

La investigación cualitativa si bien surgió, desde el nacimiento de las ciencias humanas en el siglo XIX, y se expresó en estudios investigativos en el siglo $\mathrm{XX}$, se vio opacada por el apogeo de la corriente positivista, "sin embargo, la crisis experimentada por las ciencias humanas desde los años 60 y 70 y los replanteamientos epistemológicos y metodológicos que se han realizado a partir de ella, contribuyeron a su resurgimiento" (Martínez, 2011, p. 11). A partir de estas metodologías, empiezan a reconocerse variadas formas para abordar las preguntas y los cuestionamientos que, sobre las realidades sociales, el investigador se plantea y para lo cual indaga y profundiza en lo mediato y en lo inmediato, en el reconocimiento de entornos y contextos, en el reconocimiento de lo multicultural y en las connotaciones de orden económico, social, político y ambiental, entre otros. En este marco de posibilidades, quienes nos ocupamos de la investigación, buscamos entender la perspectiva de los investigados (individuos, grupos o comunidades) acerca de los fenómenos 
que los conflictúan, para comprender sus experiencias, perspectivas, opiniones y significados, es decir, para conocer la forma en que participantes perciben subjetivamente su realidad e indagan sobre lo que realizan para transformar o mantener sus condiciones de vida.

\section{En la mirada puntual de los dos años de ejecu- ción del proyecto}

En el trabajo de campo realizado durante el año 2016, que tenía como pretensión la identificación de ciertas transformaciones en el rol de las mujeres, en razón de su vinculación a organizaciones sociales, bien pronto nos topamos con la brecha entre nuestras búsquedas y las realidades sociales que ellas habitaban, pues el encuentro con las mujeres vinculadas a las nueve fundaciones y colectivos que visitamos - tres de la costa Atlántica, tres de la costa Pacífica y tres de la ciudad de Bogotá- nos trajo de presente la crudeza de sus cotidianidades y lo aislados que estamos de ellas. Hemos de reconocer, incluso, que corrimos el riesgo de reproducir y ahondar en las exclusiones, repitiendo discursos descontextualizados, interrogando por categorías y cuestiones que no se aproximan lo suficiente a la condición de vida de las personas, familias y comunidades que sobreviven en medio de circunstancias de profundo malestar económico, político, social y cultural.

Esa escisión que reconocemos y damos por sentada, entre el quehacer intelectual y académico y las experiencias de vida de los seres humanos que, por supuesto, atienden a diversas circunstancias fenomenológicas, puede ser pasada por alto e invisibilizada. Empero, al incursionar en las comunidades, estas nos revelan complejidades y multi-determinaciones más allá de saber organizado sobre la realidad social, en la medida en que nos confronta con las perspectivas subjetivas, que como bien sabemos, tratan de ser expurgadas del discurso científico y de la producción investigativa como tal.

Nuestro proceso de trabajo de campo no fue una excepción a tal reconocimiento. Varias situaciones nos confrontaron con esa característica de la investigación de los fenómenos sociales, mencionada por diversos autores como complejidad, es decir, la cualidad dinámica y cambiante de las relaciones humanas, de los vínculos con el otro y de las dificultades para establecer conclusiones universalizadoras de las decisiones de los seres humanos, situadas en un contexto particular, "la complejidad emerge desde lo social, porque no es posible la predictibilidad del rumbo de las comunidades de seres humanos" (Salazar, 2004, p. 23). En la medida en que el devenir de las observaciones fluctúa de acuerdo a las historias de las personas y de sus particulares formas de interpretar y contar sus aconteceres, unas que cambian atendiendo a circunstancias que construyen y redefinen las historias a medida que se van narrando, "la complejidad comienza a aparecer cuando uno se plantea la pregunta por el sentido de la historia y se da cuenta que el único sentido de la historia es el que se va construyendo conforme hacemos historia" (Salazar, 2004, p. 23).

Los fenómenos y las problemáticas sociales han de ser comprendidas e investigadas desde el reconocimiento de la realidad social como una red de relaciones sociales diversas y cambiantes, y quienes nos ocupamos del ejercicio de la investigación social, estamos llamados a tratar de vislumbrar tales relaciones y determinantes para establecer aquellos factores que, en cada caso y dependiendo de la pregunta de investigación planteada, tienen mayor pertinencia y relevancia para caracterizar las interacciones de los fenómenos y los factores que dan asidero a tal complejidad. 
Ahora bien, en el método definido propiamente para el ejercicio de investigación, siguiendo la tradición de las ciencias derivada del Aristotelismo, en el Primer Libro de la Metafísica, el recorrido empieza con el reconocimiento del estado de la cuestión y se proyecta como el marco conceptual a partir del cual los análisis se irán articulando; así las cosas, la investigación se sostuvo en cuatro momentos clave. La etapa de formulación de la investigación, que nos permitió establecer los puntos de partida y la pregunta de investigación propiamente hablando, a saber: ¿Transforma la mujer su mundo privado y la visión de lo público, a partir de su participación activa en organizaciones gestadas y direccionadas por mujeres?

Tal proceso se afirmó principalmente en el ejercicio de escritura y en el debate entre las investigadoras, atendiendo a sus intereses, formación y proyección del trabajo investigativo, así como a los requerimientos institucionales que definían unos lineamientos, plazos, procesos, recursos y marco administrativo predefinido; en algún sentido, homogeneizante, pues tales presupuestos, como seguramente ocurre en otras instituciones educativas, son un marco en el que difícilmente se distinguen durante esta etapa las implicaciones y dinámicas diferenciales derivadas de asuntos tan relevantes como el tipo de investigación a realizar, la población objeto de estudio o el método elegido para el desarrollo.

Posteriormente adelantamos la etapa de diseño, en la que definimos los derroteros y justificaciones en términos del método, el tiempo, las estrategias, las técnicas, los lugares y las personas participantes en la investigación, estableciendo, por supuesto, las categorías de análisis, que en el caso de la investigación que adelantamos, fueron concretadas tras el ejercicio de revisión del estado del arte, principalmente sobre la cuestión de los cambios y permanencia en los roles sociales de las mujeres, así como de las características históricas de las organizaciones sociales de mujeres en el país. Por supuesto, establecimos las categorías de análisis fundamentales que nos permitirían definir los sentidos de la investigación, así como la información que esperábamos recabar durante la etapa del trabajo en campo. Tales categorías fueron: Rol sociocultural, Organización de la mujer, Participación de la mujer, Empoderamiento y Equidad de género.

Es claro que anticipábamos que otras categorías de análisis podrían emerger, en razón de la dinámica de la investigación cualitativa, en la medida en que es inductiva, holística, humanista, sistemática e implica una interacción entre investigadores y las personas que son causa del estudio, comprende a las personas en su propio marco de referencia, busca atender a las comprensiones y perspectivas de quienes participan en la investigación, y en especial, permite que todos los contextos y las personas participantes se constituyan como potenciales ámbitos de indagación (Ortiz, 2012, p. 17-19).

Luego, atendiendo a los tiempos y compromisos institucionales, empezamos el trabajo en campo, que inició con el establecimiento de contacto con las organizaciones que pretendíamos que hicieran parte del proceso, es decir, nos acercamos por primera vez a la realidad social, más allá de la que habíamos vislumbrado a partir de la revisión de la literatura seleccionada. Durante esta etapa, la de la ejecución, los inconvenientes más allá de las previsiones y los cronogramas empezaron a hacerse presentes: poca y errada información sobre las organizaciones sociales de mujeres reconocidas, desinterés o negativas directas por parte de las lideresas para aceptar la convocatoria a participar en la investigación -en su mayoría, debido a la 
expectativa de retribuciones económicas, al desinterés por cuenta del "manoseo" institucional y por temor a hacer visible el trabajo organizativo en razón de riesgo a la seguridad de las comunidades-, y retraso en los tiempos previstos para la realización del trabajo en campo, generado por la escasa disponibilidad de tiempo de las lideresas, la dinámica de trabajo propia de cada organización, dificultades de acceso vial, entre otras.

Superados estos obstáculos y ajustados los tiempos institucionales -el cronograma establecido contractualmente- para el inicio de la ejecución del proyecto, las investigadoras empezaron la labor de encuentro con las organizaciones, con las lideresas y las personas vinculadas, llevando como insumos las lecturas previas y los instrumentos diseñados a partir de las técnicas definidas durante el diseño: entrevistas estructuradas, entrevistas semi-estructuradas, grupos focales y registros de observación de las dinámicas relacionales.

Bastaron pocos minutos de encuentro para empezar a indagar un poco sobre las realidades de aquellas mujeres, y al hacerlo, bien pronto nos conmocionamos, pues tras el saludo de bienvenida los relatos se hicieron escuchar, como si llevaran mucho tiempo estancados, y descubrimos que aprehender la realidad de las otras y los otros, incluso desde el lugar de investigadoras y con los referentes conceptuales previstos, no nos exentaba del dolor y de la angustia, más bien, pronto nos sumergía en una confrontación que nos ponía de cara a una experiencia que acostumbrábamos situar en otros, no en nosotras mismas: la generalización de la indiferencia, esa distancia respecto a las realidades y padecimientos del otro.

Lipovetsky (2000) señaló que "las cuestiones cruciales que conciernen a la vida colectiva conocen el mismo destino que los discos más vendidos de los hit-parades, todas las alturas se doblegan, todo se desliza en una indiferencia relajada" (p. 13); en nuestro caso, de profesionales egresadas de la Universidad Nacional de Colombia, relativamente conscientes de las vicisitudes y problemáticas del país, docentes universitarias que en diversas ocasiones habían presentado a sus estudiantes lecturas, videos, ensayos y reflexiones sobre temas diversos de la cuestión social, lo que menos parecía anticiparse en el encuentro con las organizaciones, era tal confrontación, pero ocurrió. Nuestros saberes previos nos pusieron de presente que uno de los problemas que aqueja a las organizaciones sociales en el país y a las comunidades en general, no era solo la desatención del Estado, era la desatención de la sociedad civil en general, incluida la academia y sus investigadores e investigadoras. Las historias que empezamos a escuchar nos revelaron pronto que nuestros conocimientos eran, por decirlo menos, insuficientes y casi obsoletos, pues al margen del quehacer intelectual, las necesidades, problemáticas, privaciones e indiferencia ante el padecimiento de las y los compatriotas, tienen unas dimensiones que sobrepasan los efectos del mero dato estadístico.

Las condiciones de contexto, los estragos del abandono, el desplazamiento forzado, la pobreza, la exclusión, los embates de diferentes tipos de violencias y la marginalización en la que vivían las lideresas y sus comunidades, contrastaban con los esfuerzos colectivos, con los ejercicios de reconciliación, con las estrategias de afrontamiento ante el aparataje institucional, con la resiliencia y la construcción de escenarios para la consolidación de la ciudadanía y la paz que ellas desplegaban por diversos medios y con sus particulares saberes y experiencias.

Nuestro acercamiento como investigadoras se sostenía en la aceptación implícita de que 
"la finalidad primordial es la comprensión de las experiencias individuales y/o colectivas en condiciones espacio-temporales, la aceptación de la diferencia y de la singularidad de los individuos como de sus grupos, como fundamento de la tarea comprensiva" (Martínez, 2011, p. 19); no obstante, tal comprensión, la escucha necesaria durante el trabajo de campo, nos cuestionó subjetivamente y causó profundas vacilaciones y confrontaciones sobre el propósito de la investigación y de nuestro "rol", en contraste con las realidades y experiencias que nos iban siendo relatadas, nos atreveríamos a plantear que puso en tela de cuestión la "utilidad" o los "aportes" que una investigación como la nuestra podrían brindar, más allá del mero reconocimiento por la producción derivada.

Del tal titubeo emergió la necesidad de ampliar la perspectiva, pues los relatos escuchados dejaban entrever asuntos más apremiantes, más profundos y estructurales que aquellos propuestos a partir de nuestras cinco modestas categorías, por lo que definimos, ya no como lineamiento institucional sino como necesidad del proceso, la flexibilización de la aplicación de las técnicas para la recolección de la información. Además, surgieron varios interrogantes, incluso sobre el lugar de la producción académica para reescribir e interpretar la pluralidad social, en tanto se corría el riesgo de silenciar y replicar las voces de manera deliberada, al tenor de las implicaciones o lecturas que pudieran derivarse de cada "producto"; fue precisamente por esta vía que logramos cernir que nuestro interés investigativo, aunque implícito, atendía al interés de reconocer en esa complejidad, una "que señala la inexistencia de un observador neutral y puro, y que, por el contrario, el observador/concebidor debe observarse y concebirse en su propia observación" (Salazar, 2004, p. 24).

Así las cosas, más allá de los objetivos propuestos o de la pregunta de investigación proyectada, nos aprestamos a escuchar, a servir de amanuenses para aquellos relatos y por esa vía empezamos a hacer explícito que uno de los propósitos y compromisos derivados del proceso de investigación, sería el de garantizar que en la producción escrita derivada se hiciesen visibles fragmentos de sus testimonios, de sus luchas y reivindicaciones, de sus dificultades y problemáticas, todo aquello que, para quienes deambulamos por el desierto, es meramente indiferencia vaciada de sentido, una ausencia que torna lo exterior en fenómeno frío que no genera compromiso alguno (Lipovetsky, 2000, p. 37).

Surgió entonces, como asunto a desarrollar, una categoría emergente: la inmanencia ${ }^{4}$ de la guerra, fundamentalmente enfocada a establecer, de entre los relatos, las historias de vida y los esfuerzos de las mujeres de las organizaciones para hacerle frente a los embates de las violencias padecidas y propugnar por la construcción de la paz. En algún sentido, sin perder de vista los objetivos propuestos, nos ocupamos de ir entretejiendo las reflexiones en términos de la proyección, de las dificultades manifestadas e identificadas, como alternativas susceptibles de ser reconocidas por

${ }^{4}$ Utilizamos el término «inmanencia» en el sentido del: "carácter de aquella actividad o acción del agente que se da en él y que tiene en el mismo agente su propio fin, "permaneciendo", por ello, en el interior de quien la ejecuta". En otras palabras, como aquella propiedad o cualidad de una cierta realidad o fenómeno que permanece encerrada en el interior de sí misma, latente, aunque no evidente siempre, pero consustancial. Tomado de: http://www.webdianoia.com/glosario/display.php?action=view\&id=182\&from=action=search\%7Cby=I Revisado el 25/07/2016. 
la sociedad civil y por otras y otros investigadores, las circunstancias estructurales que, para las nueve organizaciones sociales participantes en la investigación, habían signado su constitución de tal forma, que no sólo se ponía en riesgo permanente su sostenimiento, sino la vida misma de las lideresas y de las mujeres vinculadas.

En esta perspectiva, aprovechamos las ventajas de la investigación cualitativa para librarnos del riesgo de reducir las historias, experiencias y relatos a meros números y cifras acumulables reveladoras de tendencias, en un intento que compartimos con muchos investigadores sociales, no sólo del país, sino de aquellas regiones que han sido recientemente azoladas por los conflictos internos y las confrontaciones armadas, quienes también tratan de ponerle rostro, historia y trascendencia social a los relatos, de modo que los futuros lectores puedan verse comprometidos con los textos que se presentan, en la medida en que son testimonios vivos, voces de resistencia, confirmaciones de empeños colectivos e individuales para hacerle frente a la exclusión, la inequidad y la muerte.

En nuestro país se destaca el trabajo realizado por el Centro Nacional de Memoria Histórica, cuyos informes, a partir del $2008^{5}$ develan trozos del horror que por cuenta de la guerra han soportado millones de colombianos y colombianas, así como historias de resistencia, reconciliación y reconstrucción, análisis y herramientas metodológicas para emprender el trabajo de la memoria, entre otras muchas publicaciones. De tal prolija producción nos permitimos destacar el Informe General del Grupo de Memoria Histórica ¡Basta Ya!, Colombia: memorias de guerra y dignidad $^{6}$, que reunió, como si de un "memorial de agravios"7 se tratase, testimonios de cientos de miles de víctimas, un esfuerzo que se sostuvo, como el de las publicaciones derivadas del proyecto de investigación que desarrollamos, en la responsabilidad ética y moral que reconocimos, como colombianas y como profesionales, con las víctimas en el país. En esta misma perspectiva, en los últimos veinticinco años, entidades estatales como el Alto comisionado para la Paz, el Ministerio del Interior y la Unidad de Víctimas, por mencionar algunas, las universidades, diferentes organismos multilaterales, fundaciones y organizaciones no gubernamentales, han producido cientos de miles de páginas acerca de la población víctima del conflicto armado interno en el país, de los factores, efectos, estragos e iniciativas de transformación ${ }^{8}$, desde perspectivas jurídicas, económicas, estadísticas, políticas, periciales y sociales.

A pesar de lo anterior, hemos de reconocer que tal esfuerzo y sus impactos aún se sostienen, a lo sumo, en los escenarios académicos y de discusión sobre las reglamentaciones políticas y jurídicas; desafortunadamente, su divulgación $\mathrm{y}$ reconocimiento entre la sociedad civil es limitado, situación que seguramente contribuye a la indiferencia de la población, principalmente urbana,

${ }^{5}$ Aludimos al Informe del caso de la masacre de Trujillo divulgado en 2008 y titulado: "Trujillo: Una tragedia que no cesa", disponible en: http://www.centrodememoriahistorica.gov.co/descargas/informes2008/informe_trujillo. pdf

${ }^{6}$ Disponible en: http://www.centrodememoriahistorica.gov.co/descargas/informes2013/bastaYa/basta-ya-colombia-memorias-de-guerra-y-dignidad-2016.pdf

${ }^{7}$ De esta forma es descrito y presentado por el Centro Nacional de Memoria Histórica.

${ }^{8}$ En una simple revisión con el buscador Google de las palabras: "Conflicto armado en Colombia siglo XXI pdf", se obtiene más de medio millón de resultados, lo que nos reafirma que la producción escrita ha sido abundante y diversa. 
sobre los hechos de guerra y confrontación armada, así como de las consecuencias y efectos que han tenido sobre todo el país. Doris Lamus (2000), a propósito de la investigación social sobre la violencia en Colombia se preguntó sobre la cuestión de "los aportes que esta enorme masa crítica de científicos sociales hace, efectivamente, en materia de encontrarle salidas a una problemática de larga data y con efectos cada vez mayores y más complejos en el tejido social, la cohesión, la confianza y la esperanza de los colombianos" (p. 2); así mismo, sobre los límites y aportes de las investigaciones que realizamos, principalmente, los docentes universitarios.

Como mencionamos antes, nuestra investigación no tuvo por objetivo indagar sobre las particularidades del conflicto armado interno, no obstante, el encuentro fue inevitable, así como aquel relacionado con las diferencias y similitudes entre las nueve organizaciones participantes, en ese sentido, el proceso tomó sus propios rumbos y los análisis se escribieron, más al ritmo de los encuentros con las mujeres que en procura del cumplimiento del cronograma, de ahí que sean muchos los hilos que quedan apenas enunciados, como insumo para nuevas investigaciones, pues un análisis como el que realizamos, más allá de la pretensión de ubicar conclusiones cerradas y temas acabados, aporta elementos que permiten mantener "al día" las reflexiones y proyecciones que han de seguirse realizando para continuar contribuyendo al conocimiento de las complejas realidades sociales que se entrelazan en los barrios y comunidades más excluidas del panorama nacional, destacando los esfuerzos de quienes son protagonistas desde las iniciativas populares y colectivas, que a su vez se proyecten para resignificar el devenir político, social y económico, y en lo posible, dinamizar y propiciar transformaciones legislativas que atiendan a las realidades identificadas.
Corroboramos que la labor investigativa en campo exige preparación, pues no está exenta de tensiones, dilemas, conflictos y riesgos, todo lo cual se exacerba por las condiciones del país, sumido en la polarización y los desacuerdos de diversos actores políticos y armados frente a la firma del Acuerdo Final para la terminación del conflicto y la construcción de una paz estable y duradera, fruto de los diálogos entre el Gobierno Nacional y las Fuerzas Armadas Revolucionarias de Colombia - Ejército del Pueblo (FARC-EP). Conocer de antemano estas circunstancias del contexto político, nos alertó sobre la importancia de asumir un proceder previsivo y cuidadoso, tanto para las personas participantes en la investigación, como para nosotras como investigadoras.

Nuestras credenciales -que supusimos, también operarían como escudos- fueron los carnés de la Institución de Educación Superior a la cual representábamos y las comunicaciones de presentación oficial, lo cual daba garantías de seguridad a las lideresas acerca de nuestra identidad y de la actividad académica que adelantábamos. La postura respetuosa frente a sus relatos, los cuales fueron transcritos de manera fiel, así como el compromiso de confidencialidad frente a las narrativas sobre las cuales nos pidieron reserva, son algunos de los aspectos a destacar, que facilitaron, a pesar de las situaciones coyunturales y de riesgo a la integridad, el encuentro con las mujeres de las organizaciones, que, además, dan cuenta de la ética asumida frente a los desempeños investigativos.

Temores y tensiones hicieron presencia en algunos territorios más que en otros, pero todos fueron asumidos con responsabilidad y desde una postura ética asertiva, lo que favoreció el desarrollo de los procesos de búsqueda de la información para su posterior análisis. La acogida por parte de las lideresas y de todas las mujeres con las cuales 
tuvimos contacto, cumplió el papel de disipador frente a las prevenciones que pudimos tener, al ir descubriendo que los esfuerzos que adelantan para convertirse en "nuevas mujeres", tanto en la escena privada como pública, exigiéndoles tenacidad, reciedumbre y empuje a toda prueba.

\section{En el momento de cierre}

Ahora bien, en el marco del cumplimiento de los productos definidos institucionalmente como sustentos de la investigación, se escriben diversos tipos de documentos: artículos remitidos a evaluación por pares académicos, ponencias presentadas en diversos escenarios, en su mayoría, también universitarios, y en nuestro caso, un libro 9 en el que nos propusimos, además de presentar los resultados de la investigación, articular las reflexiones, adaptaciones, cambios y contingencias propias del proceso realizado, como estrategia que nos permitiese convertir la obra escrita en material bibliográfico para trabajar en las aulas.

La escritura hace parte de los propósitos y fundamentos de la investigación, pues es el soporte primario de divulgación de los análisis y a la vez, contribuye al acervo bibliográfico que será tenido en cuenta para subsiguientes investigaciones. Tales productos se inscriben sin embargo en una lógica que, las más de las veces, atiende preferentemente a exigencias burocráticas de medición más que a finalidades éticas o de transformación social, a lo cual, desde luego, aspiraríamos.
La producción escrita, aunque ha aumentado sustancialmente en los últimos años, parece supeditada a cuestiones como el puntaje, la citación de los autores en otros artículos, la clasificación en categorías y los requisitos ${ }^{10}$, y no propiamente al interés de contribuir desde la investigación social, a la transformación de las dinámicas, políticas y realidades sociales, tal como lo plantea Lamus cuando afirma que:

Hay necesidad de replantear y reestructurar de manera profunda y seria el trabajo de investigación en este campo si se quiere que todo ello no sea solo el laboratorio y el observatorio permanente de los "científicos" sociales, sino el lugar donde gobernantes, estrategas, políticos, dirigentes y sociedad civil acudan en busca de las recomendaciones que se instrumentan y evalúan (2000, p. 9)

A pesar de lo anterior, consideramos que la producción escrita que nos permita, como investigadoras y como ciudadanas, acercarnos a la complejidad de las realidades sociales del país, a las historias que se siguen escribiendo y que se reescriben como fruto de la labor investigativa, y que han de proseguir a pesar de las mediciones y categorizaciones, ya que reconocemos la responsabilidad ética y social que tenemos con el país, pero además, porque el ejercicio de producción escrita implica acercarnos a otra producción bibliográfica que sirve de insumo para comprender en contexto, y articulado al devenir histórico la dinámica

9 Titulado preliminarmente: "Entre el conflicto armado y el empoderamiento de las mujeres: Nueve organizaciones colombianas", y que por dinámicas propias de la terminación de la vinculación a la institución universitaria que financió el proyecto, se encuentra en una suerte de limbo editorial, pues cuestiones como la definición de los derechos de autor y los derechos patrimoniales del financiador han dificultado la publicación del mismo.

${ }^{10}$ En la actualidad son varios los analistas de la cuestión, en su mayoría, profesores universitarios que "deben" publicar sus artículos en ciertas revistas científicas y no en otras, por exigencias que no se compadecen con los supuestos de la divulgación científica propiamente dicha; por ejemplo, el artículo: Uribe Tirado, Alejandro (2017). Publindex se usa como no se debe, Revista Alma Mater, ( $n^{\circ} 668$ ), Universidad de Antioquia, Medellín, Colombia. Disponible en: http://eprints.rclis.org/31686/1/PUBLINDEX\%20ALMA\%20MATER.pdf 
social, sus contradicciones y los compromisos que unos y otras hemos de establecer para la instrumentalización e implementación de acciones concretas que apunten a dar respuesta desde diversos lugares disciplinares, administrativos y gubernamentales a las necesidades identificadas entre la población participante en las investigaciones de esta índole.

Nuestras reflexiones, surgidas del encuentro con las lideresas y con las mujeres vinculadas a las organizaciones sociales, así como con las autoras y autores consultados a lo largo del proceso, que por el momento están consignadas en el libro que esperamos publicar pronto, tienen la intención de convertirse en herramienta y material de debate, para acercarnos con investigadores e investigadoras, con funcionarios y funcionarias, con ciudadanos y ciudadanas, a la realidad social del país, eso que apenas es esbozado en los textos, pero que palpita en las comunidades y en las calles, en los campos y en las historias de colombianos y colombianas que cotidianamente, sin mayor reconocimiento y exponiendo su integridad, insisten en transformar al país por medio de iniciativas, procesos de participación y movilización de diversa índole, (re) construyendo el tejido social, pues de él depende su supervivencia y la de sus vecinos, hijos y amigos.

En lo concerniente a las reflexiones derivadas del proyecto de investigación, es decir, al cumplimiento del objetivo del proceso, entendido como "generar conocimiento a través de la resolución del problema establecido al inicio del estudio" (Ugade y Balbastre, 2013, p. 180), y habida cuenta de las dificultades para el acercamiento a los fenómenos sociales en razón de las diversas explicaciones de las realidades experimentadas por las personas participantes en la investigación, limitadas por los recursos, los cronogramas y los plazos prescritos para la generación de resultados, es pertinente mencionar que a esta altura algunos de los discernimientos y análisis propuestos tras la culminación del proceso de investigación aparecen identificados en el apartado final bajo el título "Para no concluir".

En primer lugar, como ha sido asunto de este artículo, nos ocupamos de reflexionar a partir de las dificultades del proceso investigativo mismo, en razón de la distancia entre la urbe y la ruralidad, entre la academia y las realidades sociales; así mismo, el inesperado encuentro con la afectación del conflicto armado como una realidad latente y no como una situación histórica superada o en vía de solución, más bien, como un factor de riesgo para el desarrollo de la investigación y por supuesto para las personas participantes.

Posteriormente, nos ocupamos de presentar los procesos de cada organización, a partir de los relatos obtenidos, prestando especial atención a la articulación y consolidación de las organizaciones de mujeres, entrelazadas en algunos casos a partir de las secuelas de las historias particulares de muerte, desplazamiento, exclusión, discriminación y despojo; dicho de otro modo, en vinculación con todas aquellas situaciones que consolidan lo que varios investigadores ${ }^{11}$ designan como pobreza estructural ${ }^{12}$; presentamos entonces

${ }^{11}$ Tales como: Haughton y Khandker (2009); Ravallion (1998); Verdera (2007).

${ }^{12}$ Es decir, aquella que se enraiza en la desigualdad, las necesidades básicas insatisfechas y la acentuación y perpetuación de condiciones de exclusión para ciertos sectores de la población -con mayores efectos para las mujeres, pues persisten las diferencias de oportunidades entre los sexos y apenas se avanza en el camino hacia la equidad-, derivada, como si de un círculo vicioso se tratase, de la misma violencia. 
los procesos organizativos como iniciativas de resiliencia y resistencia colectiva ante las realidades sociales individuales, y por esa misma vía, como puentes y agentes de gestión social para el acceso y garantía de los derechos de la población vinculada. Unos esfuerzos organizativos y de gestión desde lo local, que a su vez proponen y propugnan por alternativas para la reconciliación y la superación del conflicto armado en el país.

Como puede evidenciarse en múltiples investigaciones y artículos de prensa, en informes y publicaciones de diversa índole, los embates del conflicto interno armado continúan campeando en diferentes áreas del país a manos de diversos actores, de ahí que el esfuerzo por la re construcción del tejido social, por la garantía de los derechos de las poblaciones más vulneradas sea una necesidad vigente, y una lucha que se constituye en el común denominador de la mayoría de las organizaciones sociales, a la que infortunadamente, agentes sociales y políticos diversos se oponen, condenándonos, como país, a la perpetuación de la guerra ${ }^{13}$, en un movimiento que pone en la escena pública, desde la clandestinidad, pero deambulando bajo el abrigo de la impunidad, a "pacificadores" violentos que se hacen sentir precisamente en los territorios más excluidos y desiguales. "El asesinato de líderes y defensores de derechos humanos es un fenómeno creciente que tiene en alerta a todas las organizaciones sociales, especialmente a aquellas que abanderan procesos en zonas históricamente golpeadas por el conflicto armado y en las que nuevos actores ilegales buscan establecer dominio territorial" (Osorio, 2017).
En las organizaciones sociales participantes pudimos identificar algunas características inquietantes en términos de su dinámica de funcionamiento: liderazgos solitarios, falta de operatividad de los comités de trabajo, carencia de recursos propios, escasa formación académica de algunas lideresas y sobre carga laboral no remunerada -en el caso de estas últimas-, problemáticas que podrían constituirse en ingredientes que pueden poner en riesgo la sostenibilidad de las organizaciones, $y$ quizá no sólo de las nueve que hicieron parte de nuestra investigación, sino de otras que operan en el país, como contención y refugio, como alternativa de supervivencia en vista de la desatención estatal, tanto a los derechos individuales de ciudadanos, como a las responsabilidades que el mismo Estado tiene con las organizaciones sociales y que están consagradas constitucionalmente.

En esta misma perspectiva, se hizo latente la responsabilidad y deuda histórica que tenemos respecto a las organizaciones sociales en el país, cuyos esfuerzos y aportes a la construcción de nación son invisibilizados, subalternizados, expropiados, cuando no, negados y eliminados sistemáticamente en beneficio de unos pocos acumuladores de capital, pues

El mecanismo de exclusión más directo es tal vez la violencia, cuyas motivaciones son, entre otras, la consolidación del poder político, la salvaguarda del bienestar de las élites, el control de la distribución de los recursos, la apropiación de territorios y recursos y el favorecimiento de ideologías basadas en la supremacía de una única identidad y un solo conjunto de valores (PNUD, 2016, p. 17).

\footnotetext{
${ }^{13}$ Aludimos aquí a la escabrosa realidad del asesinato sistemático de líderes y lideresas sociales y de defensores de derechos humanos, que desde el año 2018 y hasta el 30 de abril de 2019, ya asciende a 317 colombianos y colombianas asesinadas, según el estimado de Instituto Nacional de Medicina Legal. Nodal (16 de mayo de 2019). Informe revela que 317 líderes sociales fueron asesinados en los últimos 15 meses. Noticias de América Latina y El Caribe. Recuperado de: https://www.nodal.am/2019/05/ en-los-ultimos-15-meses-fueron-asesinados-317-lideres-sociales/
} 
Un proceso continuado en el que las mujeres, al menos aquellas que hicieron parte de la investigación y quienes hacen parte o lideran organizaciones de base comunitaria, fundaciones y organizaciones no gubernamentales, en general, hacen frente, a la vez que resignifican y redimensionan su lugar en la sociedad y los alcances de sus aportes a la construcción de país, más allá del umbral del rol doméstico históricamente asignado, ampliando su perspectiva en torno de la posibilidad de la participación política, de la asunción y ejercicio de poder, de la consolidación de iniciativas independientes de las decisiones de los pater familias, y en esta vía, de la transformación social de los estereotipos de género, no sólo en el marco de las prácticas de cuidado que siguen asumiendo, sino además, en los espacios de discusión comunitaria y en los territorios en los que poco a poco se han ido posicionando como protagonistas sociales, y ya no sólo como víctimas o testigos.

Finalmente, consideramos que el Estado y las Instituciones de Educación Superior deben dar pasos efectivos para el cumplimiento del postulado planteado en la Ley 30 de 1999, Título sexto - Capítulo I:

La extensión comprende los programas de educación permanente, cursos, seminarios y demás programas destinados a la difusión de los conocimientos, al intercambio de experiencias, así como las actividades de servicio tendientes a procurar el bienestar general de la comunidad y la satisfacción de las necesidades de la sociedad.

Efectivamente, evidenciamos que la docencia y la investigación son baluartes de los niveles de la educación superior, pero cumplirán su total función cuando se articulen al desarrollo y avance de la sociedad, de las regiones y de los territorios, desde donde se aportan los hechos y las realidades sociales para producir nuevos conocimientos. Tal como lo menciona la Conferencia Regional de Educación Superior en América latina y el Caribe:

Las instituciones de Educación Superior deben avanzar en la configuración de una relación más activa con sus contextos, lo cual exige impulsar un modelo académico caracterizado por la indagación de los problemas en sus contextos; la producción y transferencia del valor social de los conocimientos; el trabajo conjunto con las comunidades (CRES, 2008, p.5).

Es que cuando avancemos hacía esta aspiración afirmaremos que la educación efectivamente estimula e impulsa el desarrollo social y se dan pasos en pro de la articulación entre el saber científico y el saber popular, bajo la consideración que los aprendizajes serán mutuos. 


\section{Referencias bibliográficas}

Castañeda Berbal, E. (2002). Colombia: equidad social y educación en los años 90, UNESCO, IIPE. Recuperado de: http://unesdoc.unesco.org/images/0012/001295/129516s.pdf

Galeano Marín, M. (2018). Estrategias de investigación cualitativa: el giro en la mirada. Medellín, Antioquia. Fondo Editorial FCSH, Universidad de Antioquia.

Lamus Canavate, D. (2000). Investigación social y violencia en Colombia, Revista Reflexión Política, vol. 2 (3). Universidad Autónoma de Bucaramanga, Bucaramanga, Colombia. Recuperado de: http://www.redalyc.org/ pdf/110/11020310.pdf.

Leal, G., Malagón, E. (2006). Historia del trabajo social en Colombia: de la doctrina social de la Iglesia al pensamiento complejo. Recuperado de: http://www.humanas.unal.edu. co/tsocial/files/4112/8498/8737/HistoriaHistoria\%20del\%20Trabajo\%20Social\%20 en\%20Colombia-2.pdf.

Martínez Rodríguez, J. (2011). Métodos de investigación cualitativa. Silogismos de Investigación: Revista de la Corporación Internacional para el Desarrollo Educativo, (8). Bogotá, Colombia. Recuperado de: http:// www.cide.edu.co/ojs/index.php/silogismo/ article/view/64/53

Morin, E. (2009), Introducción al pensamiento complejo, México, D. F. Editorial Gedisa. Recuperado de: http://www.pensamientocomplejo.com.ar/docs/files/MorinEdgar_ Introduccion-al-pensamiento-complejo_ Partel.pdf

Nodal (16 de mayo de 2019). Informe revela que 317 líderes sociales fueron asesinados en los últimos 15 meses. Noticias de
América Latina y El Caribe. Consultado el 18/05/2019. Disponible en: https://www. nodal.am/2019/05/en-los-ultimos-15-meses-fueron-asesinados-317-lideres-sociales/

Ortiz, L. (2012). Curso de Investigación Cualitativa. UNAD, Bogotá, Colombia. Recuperado de: http://www.academia.edu/15022913/ Curso_Investigaci\%C3\%B3n_Cualitativa_-_Ligia_Ortiz_Zepeda_Autora_de_la_ Actualizaci\% $\% 3 \% \bar{B} 3 n$

Osorio Granados, M. (2017). Líderes sociales: los silenciados y los que resisten. Colombia 2020, El Espectador. Colombia. Consultado el 2/12/2017. Disponible en: https:// colombia2020.elespectador.com/territorio/ lideres-sociales-los-silenciados-y-los-queresisten

PNUD (2016). Panorama general. Informe sobre Desarrollo Humano 2016, Desarrollo humano para todos. Disponible en: http://hdr. undp.org/sites/default/files/HDR2016_SP_ Overview_Web.pdf.

Prats, J. (2011). Las ciencias sociales en el contexto del conocimiento científico. La Investigación en Ciencias Sociales. Universidad de Barcelona. Disponible en: http://www. ub.edu/histodidactica/images/documentos/ pdf/prats- $\% 20$ que $\% 20$ son $\% 20$ las $\% 20$ ccss. pdf

Salazar, I. C. (2004). El paradigma de la complejidad en la investigación social. Educere, Vol. 8, ( $\left.\mathrm{N}^{\circ} 24\right)$. Universidad de los Andes, Mérida, Venezuela.

Sandoval Casilimas, C. (1996). Investigación Cualitativa. Programa de especialización en teoría, métodos y técnicas de investigación social. Módulo cuarto. ICFES, Bogotá, Colombia. 